\title{
How Many Unit Equilateral Triangles Can Be Generated by $n$ Points in Convex Position?
}

\author{
János Pach and Rom Pinchasi
}

\section{INTRODUCTION.}

What is the maximum number of times that the unit distance can occur among the distances between $n$ points in the plane? This more than fifty-year-old question of Paul Erdös, published in this MONTHLY [5], opened a whole new area of research in combinatorial geometry [10].

An important variant of this problem, raised by Erdős and Leo Moser in [6], is the following. At most how many times can the unit distance occur between the vertices of a convex $n$-gon, i.e., among the distances between $n$ points in the plane in strictly convex position? Denote this maximum by $u^{\text {conv }}(n)$. Erdős and Moser noticed that $u^{\text {conv }}(n) \geq\lfloor 5(n-1) / 3\rfloor$, as is shown by a configuration containing $\lfloor(n-1) / 3\rfloor$ congruent copies of a rhombus with side length one and obtuse angle $2 \pi / 3$, rotated by small angles around one of its vertices belonging to such an angle (Figure 1). They also suggested that this bound may be sharp.

Thirty years later, Herbert Edelsbrunner and Péter Hajnal [4] came up with a better construction showing that $u^{\text {conv }}(n) \geq 2 n-7$. This is the best currently known lower bound. On the other hand, Füredi [9] proved that $u^{\text {conv }}(n)=O(n \log n)$ (see [3] for a very simple induction argument).

An equilateral triangle of side length one is called a unit triangle. The aim of this note is to show that if, instead of unit segments, we count the number of unit triangles determined by $n$ points in strictly convex position in the plane, then the maximum is attained by the ErdösMoser configuration depicted in Figure 1. We say that a set of points $S$ spans a unit triangle $T$ if the vertices of $T$ belong to $S$. Using this terminology, we have:

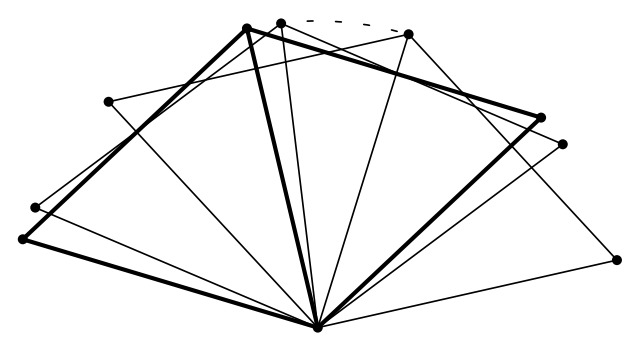

Figure 1: The Erdős-Moser configuration. 
Theorem 1. Any set of $n$ points in strictly convex position in the plane has at most $\lfloor 2(n-1) / 3\rfloor$ triples that span unit triangles. This bound is sharp for each $n>0$.

If we do not require strict convexity, we obtain a somewhat different answer. A set $S$ of $n$ points in the plane is said to be in convex position, if none of its elements is contained in the interior of a triangle with vertices in $S$.

Theorem 2. Any set of $n$ points in convex position in the plane has at most $n-2$ triples that span unit triangles. This bound is sharp for each $n>1$.

The proofs of Theorems 1 and 2 are given in Sections 2 and 3, respectively. In Section 4, we discuss the analogous questions for non-unit triangles. Our methods yield the following bounds.

Theorem 3. Any set of $n$ points in strictly convex position in the plane has at most $2 n$ triples that are the vertices of congruent copies of a fixed triangle with a given orientation.

The investigation of repeated triangles (or, more generally, simplices) in various point sets was initiated by Erdős and Purdy [7], [8]. For further results of this kind and their higher dimensional analogues, see Ábrego and Fernández-Merchant [1] and Agarwal and Sharir [2], respectively.

\section{POINTS IN STRICTLY CONVEX POSITION: PROOF OF THEOREM 1}

Throughout this section, $S$ is a fixed set of $n$ points in the plane in strictly convex position. Let $\operatorname{conv}(S)$ denote the convex hull of $S$. Connect two points $x$ and $y$ of $S$ by a straight-line segment (or edge), if $S$ spans a unit triangle one of whose sides is $x y$. If $x y z$ is a clockwise-oriented unit triangle spanned by $S$, then $x y$ is said to be a left edge with respect to $x$ and a right edge with respect to $y$. It is called a rightmost left edge with respect to $x$ if there is no left edge of a unit triangle that can be obtained from $x y$ by a clockwise rotation around $x$ through an angle smaller than $\pi$. Similarly, $x y$ is called a leftmost right edge with respect to $y$ if there is no $S$-generated right edge that can be obtained from $x y$ by a counter-clockwise rotation around $y$ through an angle smaller than $\pi$. Obviously, there is at most one rightmost left edge and at most one leftmost right edge with respect to each vertex of each unit triangle determined by $S$.

We need the following observation.

Lemma 2.1. Let $x y$ be a left edge with respect to $x$ (hence a right edge with respect to $y$ ). Then $x y$ is either the rightmost left edge with respect to $x$ or the leftmost right edge with respect to $y$.

Proof. Let $x y z$ be a clockwise-oriented unit triangle with vertices in $S$. Assume, in order to obtain a contradiction, that $x a$ is the rightmost left edge with respect to $x$ and $y b$ is the leftmost right edge with respect to $y$, for some $a \neq y$ and $b \neq x$. Then, there exist points $a^{\prime}$ and $b^{\prime}$ of $S$ such that $x a a^{\prime}$ and $y b^{\prime} b$ are clockwise-oriented unit triangles.

Let $C_{x}$ and $C_{y}$ denote the semicircles obtained by intersecting the unit circles centered at $x$ and $y$, respectively, with the half-plane containing $z$ bounded by the line $x y$. Let $y_{1}$ be the 


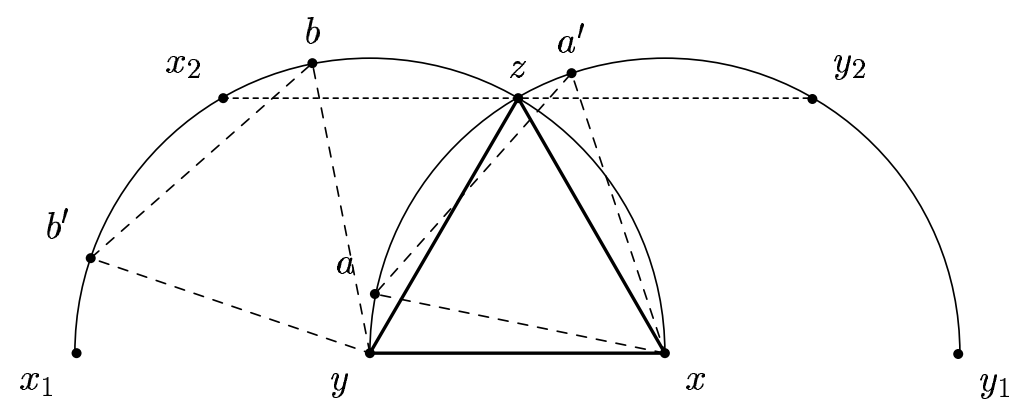

Figure 2: Illustration to the proof of Lemma 2.1.

point of $C_{x}$ diametrically opposite to $y$, and let $x_{1}$ be the point of $C_{y}$ opposite to $x$. Finally, let $y_{2}$ denote the midpoint of the arc of $C_{x}$ between $y_{1}$ and $z$, and let $x_{2}$ denote the midpoint of the arc of $C_{y}$ between $x_{1}$ and $z$ (see Figure 2).

The point $a$ cannot belong to the closed arc $\left.\widehat{\left[y_{1}, y_{2}\right.}\right]$ of $C_{x}$, because if it did, $x$ would be in $\operatorname{conv}\left\{y, a, a^{\prime}\right\}$, contradicting our assumption that $S$ is in strictly convex position. Thus, $a$ must lie on the open arc $\widehat{\left(y, y_{2}\right)}$ of $C_{x}$. This shows that either $a$ or $a^{\prime}$ must belong to the subarc $\widehat{\left(z, y_{2}\right]}$ of $C_{x}$. Similarly, either $b$ or $b^{\prime}$ must belong to the subarc $\widehat{\left(z, x_{2}\right]}$ of $C_{y}$. This places $z$ in $\operatorname{conv}\left\{x, y, a, a^{\prime}, b, b^{\prime}\right\}$, the desired contradiction.

Remark 2.2. If we make the somewhat weaker assumption that the elements of $S$ are in convex (but not necessarily strictly convex) position, then there is only one possibility: $z=a=b$.

We may assume without loss of generality that every element $x$ of $S$ belongs to at least one unit triangle spanned by $S$. Otherwise, we can discard $x$ and prove Theorem 1 by induction. Let $P_{1}$ (respectively, $P_{2}$ ) denote the set of ordered pairs $(x, e)$, where $x$ belongs to $S$ and $e$ is the rightmost left (respectively, leftmost right) edge with respect to $x$.

According to our assumption, for each $x$ in $S$ there is precisely one rightmost left edge and precisely one leftmost right edge with respect to $x$. Therefore, $\left|P_{1}\right|+\left|P_{2}\right|=2 n$. On the other hand, Lemma 2.1 implies that each side of a unit triangle contributes at least one element to $P_{1}$ or $P_{2}$. Denoting the set of unit triangles by $U$, we obtain $\left|P_{1}\right|+\left|P_{2}\right| \geq 3|U|$, whence

$$
|U| \leq \frac{\left|P_{1}\right|+\left|P_{2}\right|}{3}=\frac{2 n}{3}
$$

This bound is only slightly weaker than the statement of Theorem 1 .

To establish Theorem 1, it is sufficient to prove the following:

Lemma 2.3. There exist at least two ordered pairs $(x, y)$ such that $e=x y$ is a rightmost left edge with respect to $x$ and a leftmost right edge with respect to $y$, i.e., $(x, e)$ belongs to $P_{1}$, and $(y, e)$ to $P_{2}$.

Proof. We may suppose again that every point of $S$ belongs to at least one unit triangle. For any $x$ in $S$, let $R(x)$ signify the intersection of $\operatorname{conv}(S)$ with the open half-plane to the right of the directed line $\overrightarrow{x y}$ supporting the rightmost left edge at $x$. Similarly, let $L(x)$ denote the 
intersection of $\operatorname{conv}(S)$ with the open half-plane to the left of the directed line $\overrightarrow{x z}$ supporting the leftmost right edge at $x$. Let $\mathcal{R}=\{R(x) \mid x \in S\}$ and $\mathcal{L}=\{L(x) \mid x \in S\}$.

Notice that any minimal element of $\mathcal{R} \cup \mathcal{L}$ under containment belongs to $\mathcal{R} \cap \mathcal{L}$. Indeed, assume that, say, $R\left(x_{0}\right)$ is such a minimal element, and let $x_{0} y_{0}$ be the rightmost left edge with respect to $x_{0}$. Then $x_{0} y_{0}$ is a right edge with respect $y_{0}$. Moreover, it must be the leftmost right edge with respect to $y_{0}$, otherwise $L\left(y_{0}\right)$ would be a proper subset of $R\left(x_{0}\right)$, contradicting the minimality of $R\left(x_{0}\right)$. Thus, we have $L\left(y_{0}\right)=R\left(x_{0}\right)$, which demonstrates that the pair $\left(x_{0}, y_{0}\right)$ meets the requirements of the lemma.

Moreover, we can find a second ordered pair $\left(x_{1}, y_{1}\right)$ with the desired property by choosing a minimal element among the sets in $\mathcal{R} \cap \mathcal{L}$ entirely contained in $L\left(x_{0}\right)$. It is not hard to verify that $\left(x_{0}, y_{0}\right) \neq\left(x_{1}, y_{1}\right)$. (However, it is possible that $x_{1}=y_{0}$ and $y_{1}=x_{0}$.)

In view of Lemma 2.3, we now find that $2 n=\left|P_{1}\right|+\left|P_{2}\right| \geq 3|U|+2$, from which Theorem 1 follows.

\section{RELAXING THE CONDITION: PROOF OF THEOREM 2.}

Here we consider the case when the points are in convex, but not necessarily strictly convex, position. Consider a set of five points, $x, y, z, y^{\prime}, z^{\prime}$, with the following property: $x y z$ is a clockwise-oriented unit triangle, $x y^{\prime} z$ and $x y z^{\prime}$ are counterclockwise-oriented unit triangles. We refer to such a set as a special configuration.

The proof is by induction. For $n=3$, the assertion of Theorem 2 is trivial. Let $n>3$, let $S$ be a set of $n$ points in convex position, and assume that Theorem 2 has already been proved for sets with fewer than $n$ elements.

If $S$ has no five points that form a special configuration, then according to Remark 2.2 we can apply the argument in the last section to conclude that $|S| \leq\lfloor 2(n-1) / 3\rfloor \leq n-2$.

Thus, we may suppose that $S$ has five points $x, y, z, y^{\prime}, z^{\prime}$ forming a special configuration. It is enough to show that $S$ has a point that is incident to at most one unit triangle. Indeed, by removing such a point and applying the induction hypothesis to the remaining set, the result follows. If $y^{\prime}$ is incident to only one unit triangle, we are done. Suppose that there is another unit triangle, different from $x y^{\prime} z$, that is incident to $y^{\prime}$. It is easy to verify that the point $x_{1}$ obtained by reflecting $x$ about $y^{\prime} z$ must belong to $S$, else the second unit triangle incident to $y^{\prime}$ would have a vertex in the interior of the convex hull of the special configuration. In exactly the same way, we can argue that either $x_{1}$ is incident to only one unit triangle or the point $z_{2}$ obtained by the reflection of $z$ about $x_{1} y^{\prime}$ also belongs to $S$. This procedure must end in finitely many steps, and produce a point incident to only one unit triangle.

\section{GENERAL TRIANGLES.}

We now extend the arguments in the previous sections to general triangles. Throughout this section, let $T_{0}=x_{0} y_{0} z_{0}$ be a fixed clockwise-oriented triangle such that $x_{0} y_{0}$ is one of its 


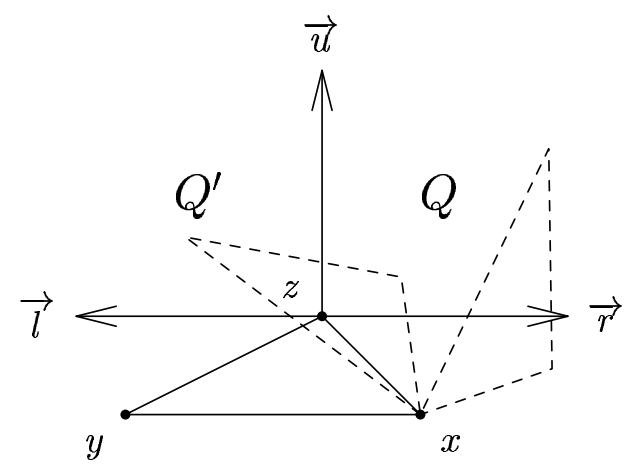

Figure 3: Definition of three auxiliary rays.

longest sides, and let $S$ be a fixed set of $n$ points in convex position in the plane. Consider a triangle $T=x y z$ congruent to $T_{0}$ whose vertices belong to $S$ and correspond to $x_{0}, y_{0}$, and $z_{0}$, respectively. Just like before, $x y$ is said to be a left edge with respect to $x$ and a right edge with respect to $y$. We say that $x y$ is a rightmost left edge with respect to $x$ (respectively, a leftmost right edge with respect to $y$ ) if there is no triangle $T^{\prime}$ congruent to $T$ that is spanned by $S$ and that can be obtained from $T$ by a clockwise rotation around $x$ (respectively, by a counterclockwise rotation around $y$ ) through an angle smaller than $\pi$.

Lemma 2.1 generalizes as follows.

Lemma 4.1. Let $T=x y z$ be a clockwise-oriented triangle congruent to $T_{0}$ whose vertices belong to a given set $S$ of points in strictly convex position, and let $x y$ be a longest edge of $T$. Then $x y$ is either a rightmost left edge with respect to $x$ or a leftmost right edge with respect to $y$.

Proof. To prove this, we need a little preparation. Suppose without loss of generality that $x y$ lies on a horizontal line and $x$ is to the right of $y$ (see Figure 3). Denote by $\vec{u}, \vec{l}$, and $\vec{r}$ the rays emanating from $z$, pointing upwards, to the left, and to the right, respectively. Let $Q$ be the convex cone (quadrant) bounded by $\vec{u}$ and $\vec{r}$, and let $Q^{\prime}$ be the convex cone bounded by $\vec{u}$ and $\vec{l}$.

Claim 4.2. Assume that $x y$ is not a rightmost left edge with respect to $x$, i.e., there is a triangle $T^{\prime}=x y^{\prime} z^{\prime}$ spanned by $S$ that can be obtained from $T$ by a clockwise rotation around $x$ through an angle $\varphi$ with $0<\varphi<\pi$. Then at least one vertex $u$ of $T^{\prime}$ must belong to the set $Q \backslash\{z\}$.

To establish this claim, let $\alpha, \beta$, and $\gamma$ denote the angles of $T$ at $x, y$, and $z$, respectively. By the maximality of $x y$, we have $\gamma \geq \alpha$ and $\gamma \geq \beta$. If $0<\varphi \leq \pi-2 \alpha$, then $z^{\prime}$ belongs to $Q \backslash\{z\}$ and we are done. If $\pi-2 \gamma<\varphi<\alpha$, then $z$ is in the interior of conv $\left(T^{\prime}\right)$, contradicting our assumption that the points are in convex position. Hence, using the fact that $\pi-2 \gamma \leq \pi-2 \alpha$, we can suppose that $\varphi \geq \alpha$. In fact, we can also assume that $\varphi>\alpha$. Indeed, $\varphi=\alpha$ implies that $y^{\prime}=z$ and $\beta=\gamma$, whence $\varphi=\alpha=\pi-2 \gamma \leq \pi-2 \alpha$, and this case has been handled before.

If $\alpha<\varphi<\pi-2 \beta$, then $z$ is in the interior of the convex hull of $x, y$, and $y^{\prime}$, contrary to the assumption that $S$ is in strictly convex position. If $\pi-2 \beta \leq \varphi \leq \pi-\alpha$, we have that $y^{\prime}$ 


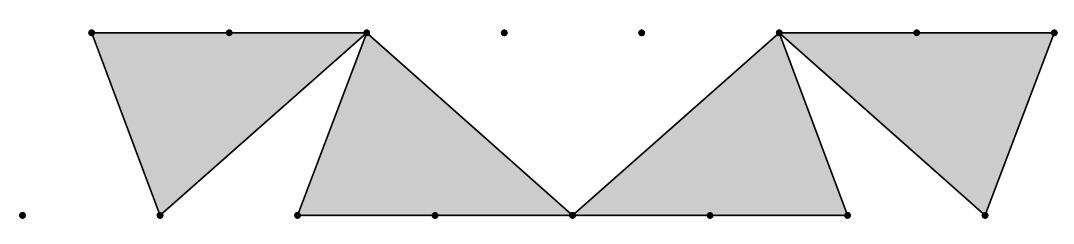

Figure 4: $n=16$ points with $2 n-8$ congruent triangles.

belongs to $Q \backslash\{z\}$. To see this, it is enough to note that the height of $T$ belonging to $x z$ is at least as large as the height belonging to the longest side, namely, $x y$.

Finally, if $\pi-\alpha<\varphi<\pi$, then $x$ must belong to $\operatorname{conv}\left\{y, y^{\prime}, z^{\prime}\right\}$, again a contradiction. This proves the claim.

By symmetry, we learn that if $x y$ is not a leftmost right edge with respect to $y$, then at least one element $u^{\prime}$ of $S$ must belong to the set $Q^{\prime} \backslash\{z\}$. Therefore, if $x y$ is neither a rightmost left edge with respect to $x$ nor a leftmost right one with respect to $y$, then we have that $z$ belongs to $\operatorname{conv}\left\{x, y, u, u^{\prime}\right\}$, which completes the proof of Lemma 4.1.

Now we are in a position to establish Theorem 3.

Proof of Theorem 3. Count the number of pairs $(p, e)$, where $p$ belongs to $S$ and $e$ is either a rightmost left edge or a leftmost right edge with respect to $p$, corresponding to the edge $e_{0}=x_{0} y_{0}$ of $T_{0}$. According to Lemma 4.1, the number of these pairs is at least the number of congruent copies of $T_{0}$ generated by $S$, that have the same orientation. On the other hand, every $p$ in $G$ belongs to at most two such pairs, so the theorem follows.

Remark 4.3. A more careful analysis of the extreme cases shows that, if $T_{0}$ is not an isosceles triangle, Lemma 4.1 and Theorem 3 remain true under the weaker assumption that the points of $S$ are in convex (but not necessarily in strictly convex) position.

Remark 4.4. In some special cases it is not hard to improve Theorem 3. Suppose, for example, that $T_{0}$ is not an acute triangle, i.e., using the same notation as in the proof of Claim 4.2, we have $\gamma \geq \pi / 2$. Assume further that $\alpha \geq 2 \beta$. Then, under the assumptions in Lemma 4.1, we can argue that $x y$ is necessarily the rightmost left edge with respect to $x$. Now every congruent copy $T=x y z$ of $T_{0}$ with a given orientation gives rise to a unique rightmost left edge at $x$. On the other hand, there is at most one such rightmost left edge incident to each vertex $v$ in $S$. Thus, we obtain:

Theorem 4. Assume that $T_{0}$ is a nonacute triangle, one of whose acute angles is at least twice as large as the other. Then any set $S$ of $n$ points in convex position in the plane spans at most $n$ copies of $T_{0}$ with a given orientation.

To see that this bound can be attained, let $v_{1}, \ldots, v_{n}$ denote the vertices of a regular $n$-gon $(n \geq 6)$ listed in clockwise order, and set $T_{0}=x_{0} y_{0} z_{0}$, where $x_{0}=v_{k+1}, y_{0}=v_{1}$, and $z_{0}=v_{k}$ for some $k$ satisfying $3 \leq k \leq n / 2$.

We conjecture that the upper bound $2 n$ in Theorem 3 can be replaced by $n$, without making any assumption on the angles of the triangle. Moreover, for nonisosceles triangles, 
this conjecture may remain true for point sets in convex (but not necessarily strictly convex) position (cf. Remark 4.3). However, it is not hard to construct a set of $n$ points lying on two parallel lines that spans $2 n-8$ copies of a suitable isosceles triangle.

\section{ACKNOWLEDGMENT}

János Pach's work has been supported by NSF grant CCR-00-98246, PSC-CUNY Research Award 63382-00-32, and OTKA-T-032452. This research was conducted while Rom Pinchasi was a Visiting Member of Courant Institute, New York University.

\section{References}

[1] B. M. Ábrego and S. Fernández-Merchant, On the maximum number of equilateral triangles I, Discrete Comput. Geom. 23 (2000) 129-135.

[2] P. K. Agarwal and M. Sharir, On the number of congruent simplices in a point set, in Proc. ACM Symposium on Computational Geometry, ACM Press, New York, 2001, pp. 1-9.

[3] P. Brass and J. Pach, The maximum number of times the same distance can occur among the vertices of a convex $n$-gon is $O(n \log n), J$. Combin. Theory Ser. A 94 (2001) 178-179.

[4] H. Edelsbrunner and P. Hajnal, A lower bound on the number of unit distances between the points of a convex polygon, J. Combin. Theory Ser. A 56 (1991) 312-316.

[5] P. Erdős, On sets of distances of $n$ points, Amer. Math. Monthly 53 (1946) 248-250.

[6] P. Erdős and L. Moser, Problem 11, Canad. Math. Bull. 2 (1959) 43.

[7] P. Erdős and G. Purdy, Some extremal problems in geometry, J. Combin. Theory Ser. A 10 (1971) 246-252.

[8] Some extremal problems in geometry IV, Congr. Numer. 17 (Proc. 7th SouthEastern Conf. Combinatorics, Graph Theory, and Computing, 1976) 307-322.

[9] Z. Füredi, The maximum number of unit distances in a convex n-gon, J. Combin. Theory Ser. A 55 (1990) 316-320.

[10] J. Pach and P.K. Agarwal, Combinatorial Geometry, John Wiley \& Sons, New York, 1995.

$$
\text { /bigskip }
$$

JÁNOS PACH is Professor of Computer Science at City College of New York, Research Professor at Courant Institute, NYU, and at Rényi Institute, Budapest. He received his Ph.D. in mathematics from Eötvös University, Budapest, in 1980 and has had visiting positions at various universities, including the University College of London, McGill University, Hebrew University, École des Hautes Etudes en Sciences Sociales. He serves on the editorial boards of five mathematical and computer science journals and has published more than 150 research papers, mostly in discrete and computational geometry and 
in combinatorics. He is a coauthor of Combinatorial Geometry, published by Wiley in 1995. He received the Lester R. Ford Award in 1990, the Rényi Prize in 1993, and the Hungarian Academy Award in 1998. Courant Institute, NYU, New York NY,USA

pach@cims.nyu.edu

ROM PINCHASI holds the position of Instructor of Applied Mathematics at the Massachusetts Institute of Technology. He ' did his undergraduate studies at the Hebrew University of Jerusalem (B.Sc., 1994). He obtained his M.Sc (1997) and his Ph.D. (2001) in mathematics from the Hebrew University of Jerusalem under the supervision of Gil Kalai, János Pach, and Micha A. Perles. His research interests include combinatorial geometry, computational geometry, graph theory, and general combinatorics.

Massachusetts Institute of Technology, Cambridge MA 02139-4307, USA

room@math.mit.edu 